Referencia para citar este artículo: Fagundez-D’Anello, D. \& González-García, D. (2018). Definir para gobernar: políticas dirigidas a niños, niñas y adolescentes en situación de calle en Uruguay. Revista Latinoamericana de Ciencias Sociales, Niñez y Juventud, 16(2), 995-1008. doi:https://doi.org/10.11600/1692715x.16223

\title{
Definir para gobernar: políticas dirigidas a niños, niñas y adolescentes en situación de calle en Uruguay*
}

\author{
DANIEL FAGUNDEZ-D'ANELLO** \\ Profesor Universidad de La República, Uruguay. \\ DIEGo GoNZÁLEZ-GARCİA ${ }^{* * *}$ \\ Profesor Universidad de La República, Uruguay.
}

\section{Artículo recibido en julio 13 de 2017; artículo aceptado en septiembre 19 de 2017 (Eds.)}

- Resumen (analítico): este artículo presenta resultados de un estudio realizado en la ciudad de Montevideo, acerca de cómo se construye la noción de sujeto de atención en las políticas dirigidas a niños, niñas y adolescentes en situación de calle. A partir de los estudios de gubernamentalidad se analizan las estrategias y técnicas de gestión del problema, desde la visión de los cargos de dirección de las políticas y de trabajadores de un proyecto que realiza la atención directa en territorio. Siguiendo un enfoque metodológico cualitativo se realiza una cartografia social con técnicas de observación participante y entrevistas semi-dirigidas. Concluimos que la definición del sujeto es el resultado de un proceso constante e inacabado, en donde intervienen una serie de elementos heterogéneos que es necesario hacer visibles.

Palaras clave: niñez, adolescencia, niños de la calle, política gubernamental (Tesauro Ciencias Sociales de la Unesco).

\section{Policies of government of the homeless childhood and adolescence}

- Abstract (analytical): This article presents the results of a study implemented in the city of Montevideo on how government policies define the subject that receives assistance, the target population for social policies for street children is constructed. After studying the policies developed

Este artículo de investigación científica y tecnológica presenta los resultados de dos investigaciones realizadas en la Maestría de Psicología Social de la Universidad de la República (UdelaR). 1-. «Cartografías de la actividad de atención directa a niños, niñas y adolescentes en situación de calle en Montevideo, Uruguay». Autor Daniel Fagundez D'Anello. Iniciado en febrero de 2014 y finalizado en marzo de 2016. Apoyada con la beca de la Comisión Académica de Posgrados de la UdelaR. Dicho proyecto estuvo adscrito en el Laboratorio Transdisciplinario de Etnografía Experimental de la Comisión Sectorial de Investigación Científica de la UdelaR; 2- «El gobierno de la niñez y la adolescencia en situación de calle. Un estudio de la racionalidad de las políticas sociales focalizadas». Autor Diego González García. Desde marzo 2014 hasta marzo de 2016. Financiada por el programa de iniciación a la investigación de la Comisión Sectorial de Investigación Científica de la UdelaR. Apoyada con la beca de maestría nacional de la Asociación Nacional de Investigación e Innovación. Es una investigación multidisciplinar, en donde se encuentran la Psicología Social, Antropología Social y la Sociología. El campo de problemas refiere a la gestión de las políticas sociales de atención a la infancia y adolescencia con vulneración de derechos.

** Docente Asistente del Instituto de Psicología Social de la Facultad de Psicología, UdelaR. Magister en Psicología Social por la Facultad de Psicología de la UdelaR. Estudiante del Doctorado de Psicología de la Facultad de Psicología de la UdelaR. Integrante del Laboratorio Transdiciplinario de Etnografía Experimental. Integrante del Núcleo Interdisciplinario Territorialidades Barriales en la Ciudad Contemporánea de la UdelaR. Inscripto en el Doctorado en Psicología de la Facultad de Psicología. Orcid: 0000-0002-6926-1853. Índice H5: 1. Correo electrónico: dfagundez@psico.edu.uy

*** Docente Asistente del Instituto de Fundamentos y Métodos de la Psicología de la Facultad de Psicología, UdelaR. Magister en Psicología Social por la Facultad de Psicología de la UdelaR y doctorando de Persona y Sociedad en el Mundo Contemporáneo de la Universidad Autónoma de Barcelona. Orcid: 0000-0002-6648-8673. Correo electrónico: dgonzalez@psico.edu.uy 
for this population, the strategies and techniques for managing the problem are analyzed through work of the people that guide the policies as well as staff that provide direct assistance in the territory. Using a qualitative methodological approach, social cartography with participant observation techniques and semi-guided interviews were carried out. The authors conclude that the definition of the subject that receives assistance is the result of a constant and unfinished process that involves a series of heterogeneous elements that more people need to know about.

Key words: homeless children, adolescents, politics and government (Unesco Social Science Thesaurus).

\section{Definir apara Governar: políticas do governo uruguaio para crianças e adolescentes em situação de rua}

- Resumo (analítico): este artigo apresenta os resultados de um estudo realizado na cidade de Montevidéu, sobre como é construída as noções de sujeito de atendimento das políticas dirigidas a crianças e adolescentes em uma situação de rua. A partir dos estudos de governabilidade, são analisadas as estratégias e técnicas de gestão do problema, a partir da visão das posições de direção das políticas e dos trabalhadores de um projeto que realiza a atenção direta no territorio. Seguindo uma abordagem metodológica qualitativa realiza-se uma cartografia social, utilizando técnicas de observação participante e entrevistas semi-direcionadas. Concluímos que a definição do sujeito é o resultado de um processo constante e inacabado, envolvendo uma série de elementos heterogêneos que devem ser visiveis.

Palavras-chave: infância, adolescência, meninos de rua, política governamental (Tesauro Ciencias Sociais da Unesco).

-1. Introducción. -2. Metodología. -3. Resultados y análisis del trabajo de campo. -4. Discusión. -5. Conclusiones. - Lista de referencias.

\section{Introducción}

Este artículo expone un análisis sobre cómo las estrategias de gobierno producen las nociones de sujeto de atención en las políticas dirigidas a niños, niñas y adolescentes (NNA) en situación de calle. El estudio fue realizado en Montevideo, Uruguay. Para abordar este problema, abrimos dos dimensiones de análisis: la primera está asociada a las formas de delimitar el sujeto de atención por parte de las políticas públicas; y la segunda comprende cómo dicha delimitación produce efectos en las acciones en un proyecto de atención directa a nivel territorial en la ciudad de Montevideo. Se realizó una revisión de los últimos documentos oficiales presentados por el Ministerio de Desarrollo Social (Mides) y el Instituto de la Niñez y la Adolescencia del Uruguay (Inau) sobre la problemática. Además, se suman dos entrevistas realizadas a personas vinculadas con la gestión del programa de atención en organismos Estatales y testimonios de los trabajadores del proyecto estudiado, que atienden directamente a NNA en situación de calle extrema.

El análisis de esta tensión se basa en los estudios de la gubernamentalidad (Castro-Gómez, 2010; Rose, 1990; Rose, O’Malley, \& Valverde, 2012). Estos conforman una red de trabajos, ensayos e investigaciones con influencia en el pensamiento de Foucault (2006), dedicados al problema de los modos en que los seres humanos somos gobernados. Estos estudios pretenden analizar la intrincada red que se pone en marcha en torno a las tecnologías de dirección de la conducta de una población. De esta manera, se adentran en la descripción de las técnicas y los procedimientos de poder, así como en cartografiar los dominios de saber implicados en las prácticas de gobierno. En este sentido, las políticas sociales pueden ser entendidas como un conjunto de estrategias dirigidas hacia la gestión de la conducta de la población, con base en unos determinados fines políticos (Fagundez-D'Anello, 2015; González-García, 2015a). Asimismo, las formas de gobierno generan una diversidad de 
autoridades, como médicos, maestros, educadores, psicólogos, así como distintos sujetos que son agentes de gobierno. Estos son agentes productores de sujetos como niños, los locos y los pobres, que son objeto de su intervención (Rose et al., 2012). Entenderemos el problema del sujeto como una cuestión fundamental para comprender la racionalidad del gobierno (Foucault, 2008; Rose, 1990). Es decir que el sujeto es todo aquello a donde se dirigen las acciones para conducir su conducta a través de nominaciones, descripciones, clasificaciones y también es todo aquello que se fuga de los dispositivos que lo pretenden controlar (Deleuze, 1990; Hacking, 2001).

Para contextualizar la producción científica y técnica sobre el tema, podemos comenzar diciendo que, en los años 80 en Latinoamérica, se hace visible el problema de los NNA en situación de calle, principalmente en zonas comerciales y urbanas, desplegándose por parte de los Estados las primeras estrategias institucionales. Según Lenta (2014), en los 90 aparecen una serie de estudios científicos que comienzan a describir, explicar, caracterizar y categorizar esa asociación entre los NNA y el problema del estar o habitar la calle, alejados de la contención familiar y de la educación.

Nieto y Koller (2015) hacen un análisis sobre el habitante de la calle, visto desde los países desarrollados y en vías de desarrollo, sumando a cómo actúa esta categoría en los NNA en situación de calle. Exponen la heterogeneidad de formas en cómo se ha categorizado este fenómeno, situando a los países desarrollados en el problema de los adultos, y en los que están en vías de desarrollo en los NNA. Con respecto a esto último, Unicef fue pionero en tomar la experiencia latinoamericana para hablar de niños «de»y «en» la calle. Los autores plantean que estas categorías son estáticas y problemáticas para la investigación. A partir de estudios en Colombia y Brasil, se ha optado por hablar de NNA en situación de calle. Esta categoría flexible incluye dimensiones como riesgo y vulnerabilidad asociadas al habitar la calle sin el cuidado de las familias. La misma está abierta a que las variables de contexto de cada territorio puedan influir en su configuración. (Lenta, 2014; Saucedo \& Taracena-Ruiz, 2011; Taracena-Ruiz, 2010; Valencia, Sánchez, Giraldo, \& Forero, 2014)

En cuanto a las relaciones humanas, la calle es un escenario proclive a que los NNA se encuentren como otros agentes de diversa procedencia que también la construyen como un espacio de habitar. En Medellín, se ha investigado sobre la relación de los NNA con vendedores ambulantes, donde existen diferentes procesos de negociación entre estos para poder compartir esas espacialidades que ambos actores los configuran de diferente manera, como por ejemplo los comerciantes ambulantes (Sánchez, Giraldo, Giraldo, \& Forero, 2016).

Las ciencias humanas y sociales aportan conocimientos que operan como instrumentos problematizadores de la situación de calle de NNA. Se identifican estudios de la psicología y de las ciencias sociales que ubican cómo algunos de los grandes problemas que producen efectos de expulsión de los NNA a la calle son, las relaciones familiares problemáticas, la vinculación de los NNA con grupos sociales vulnerables y el malestar afectivo que ambas situaciones les generan (Morales, Morales, Velázquez, Alfaro, \& Cruz, 2016). Las ciencias de la salud aportan conocimiento sobre cómo, a partir del uso de grupos focales de discusión, se pueden establecer procesos de intercambio sobre temas sensibles para los NNA en situación de calle, asociados al cuidado del cuerpo y el problema de la sexualidad. Se ha identificado que generar estos espacios promueve la participación, la aparición de preconceptos sobre el cuidado de la sexualidad, la posibilidad de introducir metodologías de las TIC para promover el interés de los NNA y aportar los conocimientos del cuidado de los profesionales de la salud para la prevención de enfermedades de transmisión sexual (Arroyave, Londoño, Valencia, Jalloh, Wylie, \& Arbeláez, 2016). Por último, se destaca que la educación ha realizado aportes valiosos en mitigar el efecto de familias expulsivas. Es el caso de la Granja-Hogar La Huella, en Uruguay, a partir de estrategia de educación no formal, de un fuerte dispositivo de inclusión social y de una contención afectiva y material, se logra mitigar los daños emocionales con los que llegan los NNA en situación de calle. Se trabaja en el aprendizaje de hábitos para la vida y la inclusión en la educación formal (Sans, 2017). La escuela y la pedagogía parecen una alianza institucional y disciplinar que 
puede promover espacios de protección, participación y promoción de estrategias de referencias positivas y cuidado para esta población (Litichever, 2016).

En las producciones científicas relevadas existen una serie de estudios que se centran en el accionar de los proyectos de atención directa a NNA en situación de calle, que aportan conocimientos sobre las prácticas de gobierno dirigidas a este sujeto (Albarrán \& Taracena, 2012; de Oña, 2010; Pérez-López, López, \& Monroy, 2011). Estas prácticas están centradas en la educación no formal y en la restitución de derechos. Su objetivo es generar estrategias para erradicar la situación de calle y propiciar espacios de protección para los NNA, tanto desde la calle, como en hogares de amparo.

Los estudios anteriormente relevados no se han centrado en las tensiones entre la producción de nociones de sujetos de atención y las formas técnicas en las que éstas son aplicadas en las políticas. Esto exige comprender las formas de saber y las estrategias de gobierno como una producción inmanente, es decir todo gobierno necesita conocer a qué y a quién se gobierna (Foucault, 2014). De esta manera, en el análisis de las políticas dirigidas a la niñez y la adolescencia podemos identificar la construcción de categorías y clasificaciones de las poblaciones, a través de instrumentos técnicos específicos. Son formas especializadas de movilizar recursos para dar respuesta al problema. Esta serie de saber-poder conlleva explícita o implícitamente una noción de sujeto de atención que debe ser analizada para comprender cómo funcionan las políticas focalizadas en NNA en situación de calle. En este sentido, entendemos que un estudio de gubernamentalidad debe tener en cuenta tres planos de análisis. Primero, sobre las tecnologías de poder, que implican la puesta en marcha de una serie de técnicas dirigidas sobre la conducta de los sujetos. En segundo lugar, saberes específicos que se producen en torno al objeto que debe ser gobernado. Por último, una determinada producción de subjetividad, que se compone por la definición de las poblaciones de atención y la emergencia de prácticas inmanentes al proceso dado entre la docilidad y la resistencia de los gobernados (GonzálezGarcía, 2015b).

Estos tres planos los podemos ver claramente en el siguiente caso en Uruguay. Nos referimos a la representación estadística de la población de NNA en situación de calle a través de técnicas de medición poblacional. El objetivo fue conocer el estado de situación para luego diseñar políticas. El primer conteo de NNA en situación de calle en Uruguay fue realizado en 2003 por una ONG. Este arrojó un resultado de 3100 en Montevideo y el área metropolitana y 4740 en el interior urbano (Gurises Unidos, 2005). El estudio presenta estadísticas inéditas en Uruguay y establece un orden de características muy detalladas del grupo estudiado. Describe prácticas concretas, distribuciones por edad, sexo, territoriales, puntos de concentración, trayectos y prevalencia de actividades según zonas.

En 2007 se realizó un segundo estudio, por el Ministerio de Desarrollo Social (Mides) y el Instituto del Niño y Adolescente del Uruguay (Inau) que replica el conteo de 2003, pero solo para Montevideo y el área metropolitana. La metodología de ambos estudios se basó en los procedimientos para censar poblaciones nómades. De esta manera, los resultados podían ser comparables y así obtener información actualizada de la población estudiada. El conteo estimó 1887 NNA en situación de calle en la zona de Montevideo y área metropolitana (Mides, 2007), un 40\% menos que en 2003. Esta disminución no aparece explicada ni analizada en la bibliografía consultada ${ }^{1}$ por lo que no podemos saber cuáles son los factores que inciden en el aumento o descenso de la población en calle. Lo que sabemos es que los procedimientos metodológicos entre los estudios comparados fue diferente y puede incidir en la diferencia. ${ }^{2}$ Aun así, la relevancia de este estudio radica en la incidencia de los resultados en la planificación de políticas sociales.

El segundo estudio arroja de forma detallada las características de los NNA y consolida distintos

$1 \quad$ Si bien es sabido que la gestión del gobierno a partir del 2005 implementó una serie de estrategias sobre las poblaciones en condiciones de pobreza, como el programa Infacalle (2005-2008) que se propuso ampliar la cobertura de atención en Montevideo y el área metropolitana de la niñez y la adolescencia en situación de calle, el informe del citado estudio no explicita ser una medición del impacto tras la implementación de dichos programas sociales.

2 Cabe resaltar que el único procedimiento de conteo que se realizó en ambos estudios comparados fue la observación, no estuvo prevista la interacción con la población para obtener información. Los observadores debían describir a través de avistamientos a distancia y completando un formulario con opciones preestablecidas. 
niveles de vulnerabilidad y riesgo de las diferentes formas de estar en la calle, que funcionan como un principio taxonómico de la población. Si bien se formulan diferentes clases o perfiles de situación de calle, estos han sido ensayados previamente desde la experiencia de los proyectos (Pérez \& Saravia, 1997; Podestá \& Lasida, 2010). La particularidad de este estudio es que reformula las políticas dirigidas a esta población, y consolida tres perfiles de NNA en situación de calle que, de algún modo, ya se encontraban operando dentro del saber profesional (Mides, 2007). Estas clasificaciones aparecen en la bibliografía consultada de forma coincidente planteando tres perfiles que representan diferentes niveles de vulneración (Podestá \& Lasida, 2010. Un primer grupo de «menor grado de vulnerabilidad», caracterizado por NNA que, localizados en su ámbito barrial, desarrollan actividades de mendicidad y recolección de forma zafral. El período de permanencia en la calle es relativamente escaso. Un segundo grupo de «nivel intermedio de vulnerabilidad», se trata de NNA alejados de su barrio de referencia, solos, en grupo de pares o acompañados por adultos que transcurren un mayor tiempo en la calle desarrollando actividades comerciales. Mantienen una relación con sus familias y con su barrio asiduamente. Sin embargo, comienzan un proceso paulatino de alejamiento. Por último, encontramos un grupo de «alto grado vulnerabilidad», también conocido como «perfil crítico» o «calle extrema». Este grupo se encuentra alejado de su familia y su barrio y hace de la calle un polo organizador de la vida cotidiana. Han desertado o han sido expulsados completamente del sistema educativo, generalmente de forma conflictiva. Su itinerario institucional transcurre entre la calle, la policía, el juzgado, cumplimiento de medidas judiciales o amparo y nuevamente la calle, un ciclo que comienza y termina en la calle. Lo han llamado circuito institucional de violencia (Fraiman \& Rossal, 2011; Pérez \& Saravia, 1997). Este perfil particular toma mayor cuerpo con base en los resultados arrojados de dicho estudio (Mides, 2007), ya que el número de NNA en esta situación de vulnerabilidad extrema ascendía a 111. Se crea una serie de dispositivos especializados para trabajar con esta población hiperfocalizada, que luego constituye la denominada Red de Atención y Protección de Niños/as y Adolescentes en Situación de Calle Extrema Inau-Infamilia (Boffa, 2011). Dentro de estos dispositivos especializados se encuentra el proyecto Farol y Revuelos, encargados de la captación y los primeros vínculos de la población en situación de «calle extrema». El artículo se centrará en la experiencia del proyecto Revuelos, para analizar cómo opera las categorías de sujeto de atención y las técnicas de abordaje implementadas. Para esto proponemos la perspectiva de los estudios de la gubernamentalidad para aportar una dimensión crítica, que a nuestro criterio ha sido poco desarrollada en Latinoamérica vinculada al problema de la infancia.

\section{Método}

El material empírico que se presentara implica el desarrollo de una cartografía social (Passos, Kastrup, \& da Escóssia, 2010; Sade, Ferraz, \& Rocha, 2013), para hacer visible los modos dinámicos en que se identifica un sujeto de atención, cómo diseñan estrategias, y cómo estas se modifican en un proceso de trabajo en territorio. El artículo se centra en los discursos de los técnicos de la gestión de las políticas y el de los operadores sociales que actúan directamente en la atención a NNA en situación de calle.

Se seleccionó el proyecto Revuelos, por ser el más nuevo de los que funciona en Montevideo y porque es uno de los resultados de los estudios estadísticos sobre la población de NNA es situación de calle realizados por el gobierno, desarrollados más arriba en el apartado de la introducción. En este sentido, resulta un caso singular en donde se puede visibilizar una racionalidad de gobierno contemporánea operando desde una diversidad de componentes estratégicos. El equipo seleccionado, se fundó en 2009 y se le asignó una zona de referencia desde el comienzo hasta el final de la Avenida 8 de octubre, una de las arterias comerciales más transitadas de Montevideo. En esta avenida y sus inmediaciones, se identificaron sesenta situaciones de calle extremas, no atendidas por ningún proyecto en funcionamiento (Infamilia-Mides, 2007). El proyecto está co-gestionado entre el Estado y la una organización de la sociedad civil (OSC), la mitad de los operadores sociales y un coordinador 
contratados por el Inau, y la otra mitad por Gurises Unidos (OSC). El mismo tiene un acta fundacional en los documentos internos de Inau, y tiene que evaluar y planificar acciones año a año. En total trabajan dos coordinadores y doce operadores/as sociales. Las edades de estos oscilan entre los veinticinco y cuarenta años, y todos están en proceso de formación o egresados en disciplinas de las ciencias sociales: psicología, trabajo social, educación social, educación popular. Un criterio que tiene este proyecto es de equilibrar la cantidad de varones y mujeres en el equipo de operadores sociales, no siendo igual en lo que es la coordinación que se elige por capacidades y experiencia según se pudo relevar en las observaciones. A esto se le integró la voz de dos gestores de las políticas que participaron en el estudio, siendo ambos de Inau y que están en la línea superior de gestión a la del proyecto seleccionado.

Las técnicas aplicadas por la cartografía se desarrollaron entre los años 2013 y 2015. Se aplicaron observaciones participantes en los diferentes escenarios de acción del proyecto (Pellicer, Vivas-Elias, \& Rojas, 2013), entrevistas en profundidad a los operadores sociales que trabajan en él, y se presentan también resultados de entrevistas realizadas a los técnicos gestores de la política (Tedesco, Sade, \& Caliman, 2013).

Los criterios de las observaciones participantes acordados con el proyecto seleccionado, fueron la participación en las reuniones de equipo semanal y de planificación y evaluación anual, la observación en recorridas por zonas de la ciudad, y los espacios de trabajo grupal con los NNA participantes del proyecto. Los criterios de las entrevistas fueron semi-dirigidas, y de las mismas, vamos a presentar los resultados asociados a la pregunta de cómo dimensionan el problema de los NNA en situación de calle, cuáles son las estrategias y técnicas asociadas al trabajo con los NNA en situación de calle, y cuál es el significado que le otorgan al NNA en situación de calle.

El alcance del estudio fue la observación participante en los diferentes escenarios de intervención del proyecto, menos la realización de entrevistas con adolescentes por implicar grandes complejidades en la obtención de los permisos éticos correspondientes. De todas formas, se autorizó a participar de actividades grupales con los adolescentes.

\section{Resultados y análisis del trabajo de campo}

La presentación de resultados la hemos ordenado, en primer lugar, dando cuenta de una indagación sobre las estrategias de gobierno implementadas en la atención a NNA en situación de calle extrema. En un segundo momento, la indagación sobre la construcción de las nociones de sujeto de atención y cómo estas han variado en los años de trabajo del proyecto seleccionado. Por último, expondremos las técnicas aplicadas en la atención directa en función de las estrategias de gobierno y los cambios en la configuración del sujeto. Consideramos que estos tres aspectos conforman una cartografía descriptiva emergente del trabajo de campo.

En cuanto a las estrategias de gobierno, una vez comenzada la actividad investigativa en el proyecto seleccionado en 2013, visualizamos que la zona de actuación no era la que previamente se había delimitado. Ante la consulta a uno de los entrevistados/as del proyecto, nos responde:

«(...) a la salida de calle de Revuelos, cuando empieza a funcionar el proyecto en 2009, se registra que no hay tales situaciones, ¿no? Por lo menos horas de permanencia en calle de mañana, de tarde, de noche... nuevamente recorridas, contactos con referentes comunitarios... o desaparecieron las situaciones o la cuantificación estuvo mal hecha. Por decir dos ejemplos y no decir ninguno de los dos (...) Gurises Unidos y el programa Calle del Inau acordaron enviar una nueva propuesta y a partir de algunas derivaciones puntuales, por ejemplo en La Teja, Malvín Norte, donde había otros proyectos, empezás a trabajar presentaciones puntuales y después empezás a trabajar en la zona y te das cuenta de que Malvín Norte estaba lleno de gurises ${ }^{3}$ que hacian calle en Hipólito Irigoyen, en Avenida

3 El término «gurí», «gurisa» «gurises» o «gurisas» es usado como un lunfardo en Uruguay para hacer referencia a los Niños, Niñas y Adolescentes. 
Italia, que estaban en el horno y eso hace abrir como..., ahi te vas anclando y vas ordenando tu mapa de intervención, lo mismo en La Teja, fuimos por una situación y empezás a hacer recorridas y permanencia en ese momento y empezaron a haber muchas situaciones.» (Coordinadores, noviembre, 2013)

En otra entrevista se plantea con mayor precisión cómo se reconfiguró la estrategia de atención a NNA en situación de calle:

«Después de haber evaluado con el equipo y las autoridades de Inau, tuvimos un mes de trabajo en donde analizamos la movilidad de la población, las razones del por qué no estaban donde habían dicho, y como llegar a sus localizaciones. Es así, que nos pusimos en contacto con la Unidad Móvil ${ }^{4}$ y Línea Azul de Inau, los cuales estaban recibiendo denuncias de situación de calle en otras zonas de Montevideo. Al llegar a esas situaciones nos encontramos que los gurises estaban desarrollando estrategias de supervivencia en calle, pero en los centros comerciales de barrios marginales, cercanos a bocas de venta de pasta base. Ante eso nos preguntamos cuál es la razón de esta movilidad y qué deberíamos hacer para poder trabajar con ellos. Y fue así que lo georreferencial dejó de ser 8 de octubre y paso a ser zonas marginales de la ciudad.» (Coordinadores de proyecto, febrero, 2014)

Tras la evidencia de que la estrategia preliminar diseñada no correspondía a la realidad, los principios que georreferenciaban el proyecto dejan de tener sentido, por lo cual su objetivo deja de ser un territorio puntual para concentrarse en las situaciones de los NNA particulares sin importar su ubicación en el mapa. Este movimiento de desterritorialización parece ser más concordante con las características nómades de la población censada por el estudio realizado en el 2007 (FagundezD’Anello, 2015).

Indagando sobre las razones del cambio del fenómeno, los entrevistados del proyecto seleccionado acuerdan unánimemente que entre el 2007, momento que realizó censo y el 2009, sucedieron eventos significativos. El más nombrado fue el de los procesos de re-alojos de familias que ocupaban asentamientos irregulares en las inmediaciones de 8 de octubre, y sus traslados a barrios periféricos de Montevideo.

Luego de años acumulados de trabajo y de la desterritorialización georreferenciar del proyecto, se cuestiona sobre si el sujeto de atención es el mismo que se categorizó en 2007, o si estos cambios hicieron que la categoría «calle extrema» ya no explique el problema. Desde los técnicos gestores de las policías, nos plantearon el siguiente:

«Estaba propuesto realizar un conteo de gurises en calle en el año 2013 pero al final no se realizó. Estoy convencido de que si bien en las zonas donde trabajan los proyectos parece que se redujo radicalmente la población en calle, ahora están relegados en otros lados como, por ejemplo, los barrios pobres y achiques ${ }^{6}$. No es que no haya más gurises en situación de calle, sino que han cambiado las estrategias por las nuevas leyes. Eso lo muestra el accionar de los proyectos que igualmente encuentran gurises haciendo calle en los barrios y no en estas zonas, lo cual marca que la situación no cambió tanto.» (Técnico gestión política, julio, 2015)

En todas las entrevistas realizadas, se puedo notar que el problema central ya no era el NNA en calle extrema asociado a la permanencia en zonas comerciales, a dormir en la calle y estar en conflicto con la ley. Al menos no solamente. Para graficar esto, vamos a referenciar a dos entrevistas diferentes a dos operadores sociales:

«Ahora nos encontramos con problemas que antes no estaban. Quizás hay menos gurises durmiendo en la calle, pero si sabemos que hay muchos que duermen en achiques, pasan

4 Dispositivo de atención itinerante a NNA en situación de calle.

5 Dispositivo receptor de denuncias ciudadanas por situación de vulneración de derechos de NNA.

6 La palabra achique forma parte del acervo verbal de los profesionales y de los NNA y hace referencia a un lugar de estancia provisoria, por lo general de características muy precarias. Suelen ser recintos ocupados y fincas precariamente construidas en asentamientos irregulares en zonas pobres de Montevideo y metropolitanas. 
horas en bocas de venta de pasta, o son reclutados en barrios periféricos para vigilar o hacer de mula. Sabemos de otros y otras que se van a vivir con personas mayores de edad, a los cuales le perdemos el rastro, y sospechamos que participan de redes informales de explotación sexual.» (Operador/a Social, junio 2014)

«Antes la policía apaleaba a los gurises en la calle, los llevaba a puerta de entrada, y si cuadraba que tenían pruebas lo judicializaban y lo metían en el Puertas. Las penas eran de dos, tres, cuatro meses, y nosotros íbamos esos meses a seguir trabajando con el gurí para pensar su salida de las medidas. Hoy con el endurecimiento de las penas a adolescentes de 14 a 17 años, en donde el mínimo es de un año, no podemos sostener todo ese periodo yendo a la Berro. Eso hizo que perdiéramos en contacto con muchos y no pudiéramos trabajar. A algunos los volvemos a ver luego de tiempo en la calle, lo cual nos hace dudar sobre si la medida privativa de libertad es eficaz o no.» (Operador/a Social, octubre, 2014) ${ }^{7}$

Ante estas problemáticas emergentes del proceso de trabajo del proyecto seleccionado, consultamos a un/a técnico/a gestor/a del proyecto sobre cómo estaban evaluando la estrategia política de intervención a esta población, y esto fue lo que nos respondieron:

«Armamos un instrumento que es un índice de vulnerabilidad muy micro...empezó siendo un formulario de cuatro preguntas sobre los gurises con los que estamos trabajando... ese instrumento no era viable porque no medía... Hicimos consultas con otros actores de otros proyectos y se colgaron a hacer un instrumento más viable. (...) lo empezamos a ver juntos y arman una ficha que marca seis variables: consumo, infracción, circulación por espacios sociales, apoyo psiquiátrico, familia, educación, las básicas... y dentro de estos puntos un desglose para ver las capacidades de los sujetos, si necesitan apoyo o no, por ejemplo, en el acceso a servicios sociales... Esto lleva a un nivel valórico que nosotros lo tuvimos que modificar porque era difícil de valorar... Por ejemplo, cuando medíamos tránsito institucional nos pasaba de ¿cómo valorar si un gurí tenía más recorrido institucional era más vulnerable o menos?... La numeración a veces te hace decir otra cosa que no es. Y después otras cosas que son complejas. Por ejemplo, ¿un gurí que está en una situación de explotación sexual está más o menos vulnerado que un gurí que tiene problemas para acceder a la cultura?» (Técnico gestión política, agosto, 2014)

Estos tres fragmentos de entrevistas muestran claramente la preocupación de los actores por comprender la transformación de una categoría y por ende del problema. En las sucesivas observaciones participantes en las reuniones de equipo del proyecto, se notó que era un tema recurrente y que todos coincidían en que cada vez se volvía más difusa la categoría consensuada en 2007. Es en ese sentido que hasta la fecha se está discutiendo sobre el sujeto y es un tema a seguir investigando en futuros estudios sobre el tema.

Para finalizar la presentación de resultados, vamos a exponer sobre cómo estos cambios, a su vez fueron transformando las técnicas utilizadas para intervenir en estas situaciones. En primer lugar, la principal técnica que se transformó fue la que el proyecto seleccionado llama captación. La captación hace referencia a la forma en la cual se detecta un caso de situación de calle en el territorio y se realizan los primeros contactos con los NNA. Se evalúa si la situación cumple con el perfil de intervención del proyecto. Consultados a qué se refiere con la noción de perfil un/a educador/a nos decía lo siguiente: «En un principio, las recorridas las realizamos en 8 de octubre, y actualmente dos por tres hacemos algunas para identificar posibles situaciones. (...) Si vemos durante dos o tres veces a un gurí que está muy repetidamente en un lugar, que su aspecto es descuidado, que está solo o con otros adolescentes en situaciones extrañas, es ahí que intentamos dar con ellos y se produce el encuentro. Es difícil de explicar, pero nosotros sabemos si es un gurí del

$7 \quad$ Puerta de Entrada» es el lugar de derivación judicial a dónde van los NNA que por alguna razón no son protegidos por sus familias. El «Puertas» es el centro de ingreso de adolescentes entre 14 y 17 años por disposición judicial entre el procesamiento por un delito. «La Berro» es el establecimiento más grande que se dedica a ejecutar las medidas privativas de libertad por disposición judicial. 
perfil o no porque nos damos cuenta la forma que tienen de moverse, de transitar la calle.» (Operador/a social, noviembre, 2014)

Sin embargo, en las observaciones participantes en territorio, visualizamos que la mayoría de los casos se construían mediante una derivación, y es en ese punto donde consultamos cómo se lograba el primer encuentro en esa situación:

«Cuando nos derivan un caso lo primero que hacemos es comunicarnos con él o la referente barrial que denuncia tal situación. Lo bueno es que cuando son vecinos, la Unidad Móvil ya tuvo los primeros encuentros y se contactó con referentes comunitarios en donde transita el gurí. En muy pocas ocasiones la situación de calle que nos encontramos no está conectada a nada. Siempre hay una escuela, un aula comunitaria, un centro juvenil (...) y tratamos de contactar al adolescente en el contexto institucional. En las pocas situaciones donde no hay ninguna institución, aplicamos la permanencia en calle y lo mismo que hacemos en las recorridas (...) tener dos o tres encuentros con los gurises y a partir de reunir la información necesaria proponer un proyecto socio-educativo (...) el proyecto se diseña en función de analizar su vínculo con la familia y ver posibilidades de una vuelta a esta, o de lo contrario pensar en el ingreso a un hogar.» (Operador/a social, julio, 2014)

Ante la pregunta sobre cuál es la metodología de trabajo en con los adolescentes ellos nombran dos:

«La proximidad es nuestra metodología. Implica en un primer momento tener un vínculo muy cercano al adolescente, para lograr ganar su confianza. Luego de lograr esto, pasamos a ser sus referentes y acompañarlos en su proceso educativo que se va construyendo según la singularidad de cada caso (...) una herramienta que nos ha ayudado mucho y que la tratamos de incorporar al trabajo es el espacio grupal (...) una vez por semana convocamos a los gurises de diferentes parejas educativas, y con dos o tres referentes del espacio se trabajan cuestiones sobre la participación de los adolescentes en el proyecto (...) arman paseos, campamentos, lo que viste la otra vez en la reunión del taller de reparar bicis, y todas las cosas que ellos quieran promover como grupo.» (Operador/a social, setiembre, 2014)

En las observaciones participantes, asistimos al espacio grupal de los adolescentes, que es la segunda metodología utilizada como principal. En este vimos como los educadores promueven diversas dinámicas lúdicas para lograr promover la socialización de los adolescentes y trabajar en pos de que ellos propongan iniciativas para llevar adelante. En una de las actividades observamos como los adolescentes, a partir de usar el mapa de la ciudad de Montevideo, marcaron los lugares a donde habían ido de paseo con los educadores y medir los kilómetros entre el lugar que fueron y de donde salieron. A partir del conteo de los kilómetros, elegirían una localidad del interior de Uruguay, para ir de paseo de fin de año. En la última observación participante que hicimos en septiembre de 2015, se había elegido un lugar, y se realizó una votación entre los adolescentes para elegir dos delegados que organizarían el viaje junto con los trabajadores del proyecto. Realizaron candidaturas a delegados, donde los candidatos debían argumentar una propuesta y se propuso una elección con voto secreto que dio como resultado a una delegada y un delegado. Es interesante cómo a través de propuestas lúdicas se recrean y simulan situaciones que funcionan como estrategias de socialización con el objetivo de trabajar la construcción de ciudadanía y la convivencia. También una re-significación del territorio de Montevideo.

\section{Discusión}

Como hemos podido ver, la construcción de un sujeto de atención y las estrategias de intervención que se diseñan en una política social, no son un proceso unidireccional entre los que diseñan y quienes ejecutan las políticas. Más bien es un proceso híbrido, compuesto por entidades heterogéneas, que traducen y transforman el sentido de las cosas, montando un curso de acción indeterminado e 
impredecible (Grau, Iñiguez, \& Subirats, 2010). Del mismo modo, los instrumentos que procuran comprender las dinámicas intrincadas de los procesos de subjetivación de una población, se vuelven representaciones que no permiten operar con certeza sobre lo que se intenta intervenir, ya que estos instrumentos de conocimiento también transforman lo que intentan conocer (Hacking, 1995; Latour, 2008).

Respecto a la construcción de las nociones de sujeto de atención definido como vulnerable, Procacci (1991) nos advierte que el gobierno de la pobreza, desde sus comienzos con la economía social, ha implicado una cierta elaboración técnica para definir una porción particular sobre la totalidad de la población. En este sentido, la cuestión de la pobreza envuelve una racionalidad de gobierno que implica definir formas de identificación y diferenciación de grupos particulares y estrategias de intervención especiales. Esta focalización repercutiría en la seguridad, circulación y libertad del resto de la población. Como señala Castel (1997), la cuestión social no es siempre la misma, ni presenta las mismas formas de abordarla y de considerarla como un problema. Los modos por los cuales se ha abordado el problema de la pobreza en estas últimas décadas, constituyen una forma específica de encargarse de la población y de comprender las prácticas. Bajo el imperativo de la reducción de gasto estatal, las políticas han tenido que sofisticar sus modos de focalización para ser más eficaces. El resultado es la priorización de los más pobres entre los pobres (Álvarez, 2011; Rojas-Lasch, 2010) y la creación de las sucesivas categorías cada vez más especializadas: NNA en situación de calle, calle extrema y NNA en situación de extrema vulnerabilidad hipervisibilizados e invisibilizados, según lo sugieren algunos planteos actuales en Uruguay (Gurises Unidos \& Inau, 2015).

Los modos de especialización en la definición de sujetos de atención por parte de las racionalidades de gobierno se traducen en la producción y en la emergencia de nuevas poblaciones y, por tanto, en un desarrollo de nuevas tecnologías y saberes encargados de su nominación y abordaje (GonzálezGarcía, 2015b). Es un dispositivo de objetivación, que identifica y clasifica prácticas, y las traduce en poblaciones focalizadas, de tal manera que estas últimas se vuelvan inteligibles y puedan ser conducidas en función de determinados fines. Cabría preguntarse aquí ¿cuáles son los efectos a largo plazo sobre los sujetos gobernados de estas objetivaciones? Para esto sería deseable en futuras investigaciones enfocarse en los testimonios de los adolescentes implicados.

La clasificación de la población en categorías como «vulneración social» o en «riesgo social» o, más específicamente, los «NNA en situación de calle extrema» son construidas por saberes que proporcionan un marco de inteligibilidad de los sujetos que se intenta gobernar. Es decir que las formas de gobierno necesitan de una cierta construcción de conocimiento sobre lo que se quiere gobernar (Rose, 1997). El ejercicio de las categorías interactúa con los sujetos a partir de una red institucional de gobierno que modela las conductas, determina ciertas posibilidades de acción y un modo de existencia particular. Este proceso no es unidireccional, sino que es interactivo, ya que las clasificaciones conllevan nuevas posibilidades subjetivas de experimentarse y de vivirse, transformando las formas de ser, pensar y sentir. Como consecuencia, dichas transformaciones modificarán los criterios de clasificación anteriores y se crearán nuevas categorías para nombrar dichos cambios. Este proceso es descripto por Hacking (1995) como «efecto bucle» para señalar el proceso interactivo en la construcción de categorías humanas y los sujetos. Este efecto coloca a las categorías en proceso inestable y en constante tensión. Si tomamos en cuenta la forma en que se ha definido el problema en Uruguay, la emergencia del proyecto que hemos analizado aquí y los procesos de redefinición de las nociones de sujeto atención que han hecho las políticas, podemos afirmar lo siguiente: la transformación de las diferentes nociones de sujeto de atención conlleva un cambio en las técnicas de intervención y los procesos de la gestión del problema, siendo estas afectantes en las formas de re-definición del problema.

\section{Conclusiones}

El artículo presenta resultados sobre los procesos por los cuales se construyen y definen las nociones de sujeto de atención, a partir de la realización de una cartografía social de un proyecto 
especializado en la atención de la niñez y la adolescencia en situación de calle en la ciudad de Montevideo. Toda práctica de gobierno define sujetos, dispone una serie de técnicas para dirigir su conducta y depende de una producción de conocimiento que lo estudian y enuncian. Las formas de caracterizar, describir, estimar cantidades y concentraciones, georreferenciar trayectorias y prácticas, van moldeando a un sujeto de gobierno cada vez más definido y focalizado como efecto de la especialización y sofisticación de las políticas. Estos procesos no son abstractos, sino que interactúan con los individuos de tal manera que, tanto estos como las categorías que los nombran, resultan modificados (Hacking, 1995). De este modo, es posible estudiar la construcción del sujeto, siguiendo las estrategias por las cuales las poblaciones son gobernadas. Tales estrategias de gobierno moldean un sujeto y un cierto campo empírico para dirigir o conducirlo hacia determinados fines. A partir de esto, se analizaron los procedimientos, las estrategias y las tácticas de gobierno dirigidas a NNA en situación de calle. Entenderemos aquí que las tecnologías de gobierno presentan una particularidad estratégica con respecto a la producción de subjetividad. Por un lado, despliegan estrategias de sujeción vinculadas a la normativización y disciplinamiento de los cuerpos de los individuos. Por otro lado, incluyen estrategias de subjetivación, que tienen en cuenta la capacidad de los gobernados para auto concebirse libremente, considerando sus deseos y sus formas particulares de ser (Castro-Gómez, 2010). Para comprender estas estrategias y su racionalidad política es conveniente vincularlas, por un lado, con una cierta forma de definir el problema y, por otro, con la persecución de determinados objetivos y fines. Estos dos aspectos fundamentan y sostienen la construcción de técnicas de dirección de la conducta de los sujetos.

Sin embargo, la producción de subjetividad no se reduce solo a los efectos de los mecanismos técnicos del poder ni a los enunciados producidos por los dominios de saber. La subjetividad es lo que siempre se fuga de los dispositivos, ya que estos no pueden capturarla en su totalidad (Deleuze, 1990). Esta incompletud de los dispositivos en el tratamiento del sujeto refiere por lo menos a dos aspectos. En primer lugar, la construcción de clasificaciones humanas supone procesos de interacción entre los individuos categorizados y las categorías que los nombran, ambos son modificados creando un efecto bucle (Hacking, 1995, 2001). Estos efectos subjetivos interpelan las formas de saber creando efectos inesperados que conllevan a reformulaciones y ajustes en las herramientas técnicas de categorización. Al decir de Butler (2002), los cuerpos, en última instancia, siempre se resisten a las nominaciones que pretenden nombrarlos. En segundo lugar, las tecnologías de gobierno contemporáneas no persiguen la docilidad de un sujeto matrizado de forma homogénea, sino que promueven nociones como la autonomía y la libertad (Foucault, 2006, 2007; Rose, 1990, 1997). De esta manera, las políticas funcionan más como una transferencia de herramientas para la gestión y administración individualizada de un sujeto ciudadano responsable de sí mismo, que como una forma de dominación totalizante. Por estas razones, comprendemos que la subjetividad se escurre por los dispositivos, presionándolos, agrietándolos y erosionándolos en todo momento. Toda definición homogénea del sujeto encuentra dificultades técnicas.

\section{Lista de referencias}

Albarrán, G., \& Taracena, B. E. (2012). Análisis de implicación de educadores de niños y niñas en riesgo de calle: el trabajo en una organización de asistencia social en la Ciudad de México. Revista Latinoamericana de Ciencias Sociales, Niñez y Juventud, 10(2), 957-970.

Álvarez, S. (2011). Gubernamentalidad neoliberal y focopolítica en América Latina: los programas de transferencia condicionada. ¿Políticas de cohesión social sobre los pobres? En C. Barba-Solano, \& N. Cohen (Eds.), Perspectivas críticas sobre la cohesión social: desigualdad y tentativas fallidas de integración social en América Latina, (pp. 251-281). Buenos Aires: Clacso.

Arroyave, D. C., Londoño, S. P., Valencia, N. G., Jalloh, C., Wylie, J., \& Arbeláez, C. A. R. (2016). Grupos focales de discusión: estrategia para la investigación sobre salud sexual con adolescentes

Rev.latinoam.cienc.soc.niñez juv 16 (2): 995-1008, 2018 
con experiencia de vida en calle en Medellín, Colombia. Facultad Nacional de Salud Pública, 34(3), 285-296.

Boffa, J. (2011). Red de Atención y Protección de Niños/as y Adolescentes en Situación de Calle Extrema. Montevideo: Inau-Infamilia.

Butler, J. (2002). Cuerpos que importan: sobre los límites materiales y discursivos del «sexo». Barcelona: Paidós.

Castel, R. (1997). Las metamorfosis de la cuestión social : una crónica del salariado. Buenos Aires: Paidós.

Castro-Gómez, S. (2010). Historia de la gubernamentalidad: razón de estado, liberalismo y neoliberalismo en Michel Foucault. Bogotá, D.C.: Siglo del Hombre Editores, Pontificia Universidad Javeriana, Pensar, Universidad Santo Tomás.

de Oña, J. M. (2010). Relaciones educativas de acompañamiento de un equipo de educadores de calle: evaluación de un proyecto de intervención socioeducativa en barriadas marginales. Revista de Ciencias de la Educación: órgano del Instituto Calasanz de Ciencias de la Educación, (222), 135-148.

Deleuze, G. (1990). ¿Qué es un dispositivo? En M. Foucault, Filósofo. (pp. 155-163). Barcelona: Gedisa.

Fagundez-D'Anello, D. (2015). Cartografías de la actividad de atención directa a niños, niñas y adolescentes en situación de calle en Montevideo, Uruguay. Psicología, Conocimiento y Sociedad, 5(1), 25-53.

Foucault, M. (2006). Seguridad, territorio, población: curso en el Collége de France (1977-1978). Buenos Aires: FCE.

Foucault, M. (2007). Nacimiento de la biopolítica: curso en el Collége de France (1978-1979). Buenos Aires: Fondo de Cultura Económica.

Foucault, M. (2008). Tecnologías del yo y otros textos afines. Buenos Aires: Paidós.

Foucault, M. (2014). Del gobierno de los vivos, curso en el Collège de France (1979-1980). Buenos Aires: Fondo de Cultura Económica.

Fraiman, R., \& Rossal, M. (2011). De calles, trancas y botones: una etnografía sobre violencia, solidaridad y pobreza urbana. Montevideo: División Programas y Proyectos de la Subsecretaría del Ministerio del Interior.

González-García, D. (2015a). El gobierno de la niñez y la adolescencia en situación de calle: un estudio de la racionalidad de las políticas sociales focalizadas. Recuperado de: https://www. colibri.udelar.edu.uy/handle/123456789/7563

González-García, D. (2015b). El gobierno de la niñez y la adolescencia en situación de calle. En M. De Martino (Coord.), ¿Familias Contemporáneas-Intervenciones Contemporáneas?, Familias y nueva matriz de protección social. (pp. 155-167). Montevideo: Universidad de La República. Recuperado de:

http://cienciassociales.edu.uy/departamentodetrabajosocial/wp-content/uploads/sites/5/2015/10/ Coloquio-Familia-documento-1.pdf

Grau, M., Iñiguez, L., \& Subirats, J. (2010). La perspectiva sociotécnica en el análisis de políticas públicas. Psicología Política, (41), 61-80.

Gurises Unidos. (2005). Niños, niñas y adolescentes en situación de calle en Uruguay ¿Cuántos son? Recuperado de: http://www.gurisesunidos.org.uy/

Gurises Unidos-Inau. (2015). No soy de aquí, ni soy de alla... Montevideo: Gurises Unidos-Inau.

Recuperado de:

http://www.gurisesunidos.org.uy/wp-content/uploads/2015/09/Proyecto-Revuelos1.pdf

Hacking, I. (1995). The looping effects of human kinds. En D. Sperber, D. Premack, \& J. Premack (Eds.), Causal cognition: A multi-disciplinary debate. Oxford: Oxford University Press.

Hacking, I. (2001). ¿La construcción social de qué? Barcelona: Paidós. 
Infamilia-Mides. (2007). Todos contamos: estudio de dimensionamiento de la situación de calle de niños, niñas y adolescentes. Montevideo: Mides.

Latour, B. (2008). Reensamblar lo social: una introducción a la teoría del actor-red. Buenos Aires: Manantial.

Lenta, M. M. (2014). Niños y niñas en situación de calle: territorios, vínculos y políticas sociales. Revista de Psicología, 22(2), 29-41. Recuperado de: https://doi.org/10.5354/0719-0581.2013.30851

Litichever, C. (2016). Entre la calle, la escuela, y las instituciones: trayectorias institucionales de jóvenes. Revista Latinoamericana de Ciencias Sociales, Niñez y Juventud, 14(1), 177-190. Recuperado de: https://doi.org/10.11600/1692715x.1411111014

Mides. (2007). Estudio de dimensionamiento de la situación de calle de niños, niñas y adolescentes. Montevideo: Mides.

Morales, K. D., Morales, L. M. F., Velázquez, L. P., Alfaro, R. de M. S., \& Cruz, P. L. (2016). La familia, malestar afectivo y redes sociales en niños y adolescentes en situación de calle. Revista Salud, Historia y Sanidad, 11(2), 29-50. Recuperado de: https://doi.org/10.1907/shs.112.167

Nieto, C. J., \& Koller, S. H. (2015). Definiciones de habitante de calle y de niño, niña y adolescente en situación de calle: diferencias y yuxtaposiciones. Acta de Investigación PsicológicaPsychological Research Records, 5(3), 2162-2182.

Passos, E., Kastrup, V., \& da Escóssia, L. (2010). Pistas do método da cartografia: pesquisaintervenção e produção de subjetividade. Porto Alegre: Sulina, Meridional.

Pellicer, I., Vivas-Elias, P., \& Rojas, J. (2013). La observación participante y la deriva: dos técnicas móviles para el análisis de la ciudad contemporánea. El caso de Barcelona. Eure, 39(116), 119139. Recuperado de: https://doi.org/10.4067/S0250-71612013000100005

Pérez, D., \& Saravia, C. (1997). El trabajo del educador en las diferentes áreas de abordaje. Revista Nosotros, 1(6), 32-34.

Pérez-López, R., López, R. P., \& Monroy, M. Á. A. (2011). Identidad y práctica profesionales del educador y la educadora de calle en México. Revista Latinoamericana de Ciencias Sociales, Niñez y Juventud, 7(2). Recuperado de:

http://revistaumanizales.cinde.org.co/index.php/Revista-Latinoamericana/article/view/197

Podestá, M., \& Lasida, J. (2010). Herramientas para el abordaje integral de niños, niñas y adolescentes en situación de calle. Montevideo: Gurises Unidos, Telefónica.

Procacci, G. (1991). Social Economy and the Goverment o Poverty. En G. Burchel, C. Gordon, \& P. Miller (Eds.), The Foucault Effect: Studies in governmentality, (pp. 151-168). Illinois: The University of Chicago Press.

Rojas-Lasch, C. (2010). Gobernar la extrema pobreza: un análisis del dispositivo de intervención Chile Solidario-Puente. En V. Lemm (Ed.), Michel Foucault: neoliberalismo y biopolitica, (pp. 51-83). Santiago: Universidad Diego Portales.

Rose, N. (1990). Governing the soul: The shaping of the private self. London-New York: Routledge.

Rose, N. (1997). El gobierno en las democracias liberales «avanzadas»: del liberalismo al neoliberalismo. Archipiélago: Cuadernos de Crítica de la Cultura, (29), 25-40.

Rose, N., O’Malley, P., \& Valverde, M. (2012). Gubernamentalidad. Astrolabio, 0(8). Recuperado de: http://revistas.unc.edu.ar/index.php/astrolabio/article/view/2042

Sade, C., Ferraz, G. C., \& Rocha, J. M. (2013). O ethos da confiança na pesquisa cartográfica: experiência compartilhada e aumento da potência de agir. Fractal: Revista de Psicología, 25(2), 281-298.

Sánchez, D. M., Giraldo, A., Giraldo, Á., \& Forero, C. (2016). Compartir y desconfiar: mirada de los venteros acerca de los niños en situación de calle. Revista de Salud Pública, 18(3), 391-403. Sans, I. (2017). Granja-hogar La Huella para niños y adolescentes: fusión metodológica de involucramiento radical. Revista Latinoamericana de Ciencias Sociales, Niñez y Juventud, 15(1), 281-293. https://doi.org/10.11600/1692715x.1511731062016

Rev.latinoam.cienc.soc.niñez juv 16 (2): 995-1008, 2018 
Saucedo, I. A., \& Taracena-Ruiz, B. E. (2011). Habitar la calle: pasos hacia una ciudadanía a partir de este espacio. Revista Latinoamericana de Ciencias Sociales, Niñez y Juventud, 9(1), 269-285.

Taracena-Ruiz, E. (2010). Hacia una caracterización psico-social del fenómeno de callejerización, $8(1), 393-410$.

Tedesco, S. H., Sade, C., \& Caliman, L. V. (2013). A entrevista na pesquisa cartográfica: a experiência do dizer. Fractal: Revista de Psicologia, 25(2), 299-322.

Valencia, B, J., Sánchez C, J., T, M., C, L., Giraldo P, Á., \& Forero P, C. (2014). Homeless children: A permanent risk. Revista Facultad Nacional de Salud Pública, 32(2), 85-91. 